\title{
Les manuscrits de travail des linguistes : un nouveau champ d'investigation génétique
}

\section{Irène Fenoglio}

\section{(2) OpenEdition}

1 Journals

\section{Édition électronique}

URL : http://journals.openedition.org/genesis/817

DOI : $10.4000 /$ genesis. 817

ISSN : 2268-1590

\section{Éditeur :}

Presses universitaires de Paris Sorbonne (PUPS), Société internationale de génétique artistique littéraire et scientifique (SIGALES)

Édition imprimée

Date de publication : 15 novembre 2012

Pagination : 7-10

ISBN : 978-2-84050-869-4

ISSN : 1167-5101

\section{Référence électronique}




\title{
Les manuscrits de travail des linguistes: un nouveau champ d'investigation génétique
}

\author{
Irène Fenoglio
}

\section{Un champ herméneutique}

$C$

omment travaille un linguiste? Quelles sont les activités sur lesquelles se fonde sa puissance d'invention? Le linguiste laisse apparaitre dans ses notes et ses brouillons toutes les sollicitations méthodologiques par lesquelles il passe, les hésitations auxquelles il est en proie, les calculs terminologiques et les prescriptions, voire injonctions qui en résultent en vue de la finalisation de son discours adressé à ses pairs et destiné à enrichir le savoir et le développement théoriques. Le texte publié, référable, condamne toutes ces élaborations à l'invisibilité, sinon à l'inexistence, alors même qu' elles construisent et explicitent le processus de théorisation.

Le généticien linguiste les débusque, les analyse et, par là même, inscrit son travail dans le mouvement d'enrichissement des connaissances visé par les textes dont il met en valeur l'avant-texte. De ce fait, la lecture de manuscrits de théorie linguistique implique d'emblée une recomposition interprétative de la lecture du texte correspondant publié.

Ce nouveau champ d'investigation génétique s'inscrit dans une complexité très particulière. Des outils à l'objet d'observation, on y circule dans un même matériau : la langue, la langue dans son fonctionnement systémique et dans son emploi en discours. La tâche d'analyse en est d'autant plus difficile. "La langue peut servir à sa propre description " ${ }^{1}$, note Benveniste, elle est en effet le seul élément qui se dit dans son propre matériau; il ajoute: "les systèmes sémiotiques autres que la langue ne se suffisent pas à eux-mêmes et ont tous besoin de la verbalisation, pour cette raison d'abord que seul est signifiant ce qui est dénommé par le langage ${ }^{2} »$.

Inaugurée avec les manuscrits de Saussure (années cinquante), la mise en valeur des sources manuscrites des linguistes vient d'élargir son champ d'investigation. De l'utilisation des manuscrits à seules fins éditoriales avec Saussure, la recherche, depuis ces cinq dernières années, se tourne vers une exploitation résolument génétique, initiée, elle, avec le fonds Benveniste de la BnF, mais se développant dans la prospection d'autres archives de linguistes et leur exploitation génétique.

Le présent numéro de la revue Genesis rend compte de ce mouvement par lequel un nouveau champ se constitue. Première publication d'ensemble, il propose, en quelque sorte, un état des lieux sur les fonds de linguistes et sur les possibilités de leur exploration. Rien

1. Note préparatoire au Cours 15 du 24 mars 1969 au Collège de France (BnF, Pap or, boîte 40, env. 80, fo 201).

2. Note préparatoire au Cours 5 du 13 janvier 1969 au Collège de France (BnF, Pap or, boîte 40, env. 80, fo 38). 
n' existe, à ce jour, sur ce sujet, ni en France, ni ailleurs. Cela légitime l' enthousiasme, mais engage, dans le même temps, une responsabilité certaine.

Nous sommes bien face à un champ qui se constitue. Il a son terrain : les archives et manuscrits de linguistes ; il a ses outils : l'analyse linguistique, la génétique du texte, et l'historiographie linguistique ; il a son domaine : histoire des idées, processus de genèse, constitution de concepts. Par ailleurs, dans le même temps qu'il se constitue, ce champ ouvre lui-même l'inventaire critique de ses pratiques et de ses problématiques.

Pourquoi l'intérêt pour les fonds linguistiques s'est-il déclaré si tardivement? D’un côté, les linguistes généticiens se sont traditionnellement consacrés exclusivement à des corpus littéraires, soucieux d'apporter la rigueur d'analyse de leur discipline à l'interprétation des processus scripturaux, jusqu'à l'ouverture du fonds Benveniste qui a mobilisé un tout petit nombre d'entre eux. D'un autre côté, il est de fait que les travaux linguistiques, y compris les études d'épistémologie linguistique, n'ont jamais manifesté beaucoup d'intérêt pour l'analyse génétique et il faudra du temps avant que l'historiographie linguistique intègre à ses données les résultats des analyses génétiques. Cela ne doit pas nous empêcher d'engranger. Il est clair que l'observation de l'écriture en production, en train de se nouer sur une suite de notes et brouillons, ne peut qu'être un facteur de développement des résultats obtenus par l'analyse des textes finis ${ }^{3}$.

Dans le présent dossier, on le remarquera aisément, la part belle est faite aux manuscrits de Saussure : trois articles lui sont entièrement consacrés (Matsuzawa, Sofía, D'Ottavi) et un quatrième article exploite plusieurs de ses manuscrits (Fenoglio). Il y a une double logique historiographique à cette présence marquée : d'une part, Saussure est considéré comme le "premier linguiste ", fondateur de la discipline et, d'autre part, le fonds Saussure est le premier à avoir été ouvert. S’ajoute à cette logique le fait que quelques jeunes spécialistes de Saussure qui ont fait eux-mêmes la démarche de se former à la méthodologie génétique s'efforcent, aujourd' hui, d'analyser les manuscrits du linguiste non seulement à des fins éditoriales mais aussi pour y introduire le point de vue génétique. Ces travaux réactivent la confrontation entre philologie et génétique, au profit, semble-t-il, de la génétique, qui permet l'ouverture de diverses problématiques, notamment celles d'ordre épistémologique. Il est notable que les articles consacrés ici à Saussure ouvrent trois thématiques différentes et permettent de mettre en valeur un fonds, encore peu connu par rapport à celui de la Bibliothèque de Genève, celui de la bibliothèque de Harvard (D'Ottavi). Tous les articles sur Saussure font lien entre eux et trouvent une cohérence avec l'entretien que D'Ottavi a mené avec Tullio De Mauro.

Benveniste dont, nous le rappelons, le fonds a été le premier à avoir été abordé génétiquement, fait l'objet de deux contributions : une étude génétique particulière sur son article «La blasphémie et l'euphémie »(Ono) et un article qui s'appuie sur l'exploitation approfondie (bien que loin d'être achevée) de l'ensemble du fonds de la BnF pour exposer les problèmes que pose la genèse du geste linguistique (Fenoglio).

Par ailleurs, ce numéro ouvre, littéralement pour la première fois, des fonds d'autres linguistes : Lucien Tesnière (Chepiga, Muni Toke), Louis Hjelmslev (Badir), Gustave Guillaume (Tollis), Antoine Meillet (Fenoglio, Puech).

3. Pour plus de détails sur le développement récent de ce champ, voir, dans ce numéro, l'article « Genèse du geste linguistique : une complexité heuristique ». 


\section{Des terrains d'investigation diversifiés}

Ce numéro de Genesis fait apparaître non pas tant de nouveaux thèmes génétiques que de nouveaux points de vue sur des questions déjà abordées par la génétique littéraire comme, par exemple, la correspondance, les différents genres de textes, la genèse éditoriale, les processus métascripturaux, etc.

Pour dégager les caractéristiques propres à ce nouveau champ, il faut aussi en faire apparaître les difficultés quant à la découverte des fonds, à la diversité de l'état de ces fonds, à leur accessibilité et quant à la nouveauté que cela implique de travailler génétiquement ce domaine. Plusieurs questions sont ainsi abordées : genèse de textes théoriques mais aussi édition, traduction, correspondance, préparation de textes ou d'ouvrages non achevés. Le statut épistémologique de chacune de ces thématiques les constitue en terrain, prenant place dans un ensemble où pourra avoir lieu la confrontation avec d'autres points de vue que le point de vue génétique pour la compréhension du fait linguistique:

- la genèse des notions et concepts et la description du processus même de conceptualisation; presque tous les articles approchent cette question, mais certains en traitent directement, sinon en exclusivité (Fenoglio, Matsuzawa, Chepiga) ;

- la fonction essentielle, en genèse des idées théoriques, des comptes rendus scientifiques. E. Sofía et V. Muni Toke montrent l'intérêt des brouillons de comptes rendus (l'un de Saussure, l'autre de Tesnière) qui permettent de découvrir les questions sousjacentes que les auteurs, par prudence, ne conserveront pas. Sofía montre chez Saussure l'écart entre la reprise presque ininterrompue d'une argumentation de plus en plus puissante et généralisante sur l'espace du brouillon et l'argumentation raisonnable qui sera, au final, transmise aux pairs. Cela constitue un véritable enjeu : la nécessité d'aller regarder comment l'argumentation scientifique s'élabore, quelle puissance d' exaltation elle doit d'abord engendrer et inscrire sur papier avant de ne divulguer que ce qui sera utile pour la discussion savante. G. D'Ottavi analyse les différentes réécritures d'un compte rendu non linguistique de Saussure pour en montrer l'intérêt pour la compréhension de ce qu'était un linguiste à l'époque et la façon dont il fonctionnait : une culture "générale » immense, un savoir multispécialisé et surtout, une connaissance approfondie - et absolument essentielle - du sanskrit sans lequel il n'y aurait pas eu d'étude de l'indo-européen, puis de grammaire comparée qui, elle-même, a permis le passage à la linguistique générale;

- l'intérêt d'analyser les échanges scientifiques que l'on peut trouver dans la correspondance entre les linguistes, comme le montre V. Muni Toke avec l'échange entre Tesnière et Pichon, ou comme le montre Marc Décimo avec l'échange entre Saussure et Duvau, celui-là corrigeant celui-ci;

- la richesse des notes, plans, brouillons, que l'on rencontre en ouvrant les dossiers de préparation de textes ou d'ouvrages qui n'ont pas abouti à un texte pouvant être considéré comme définitif ; ainsi le lourd dossier de "De l'essence double du langage » de Saussure (Matsuzawa), ou cet ensemble préparatoire à un ouvrage que Tesnière aurait pu intituler «La glottologie » (Chepiga) ;

- l'importance des problèmes de traduction, comme le montre S. Badir à propos de l'édition de La Structure fondamentale du langage de Hjelmslev; 
- les questions qui se posent pour identifier et comprendre les différentes éditions d'un même texte comme celles de "Observation et explication dans la science du langage » de Gustave Guillaume (Tollis) ;

- enfin, une des questions les plus épineuses : comment éditer un contenu de discours présent sur des manuscrits sous forme d'un texte lisible? C'est toute la question de l'édition (sous le titre Écrits de linguistique générale) des manuscrits de Saussure portant sur «De l'essence double du langage » (Matsuzawa).

Deux entretiens viennent, en quelque sorte, encadrer ce tableau heuristique. Tullio De Mauro (interrogé par G. D’Ottavi) remet en situation les premières approches des sources manuscrites de Saussure et le travail de traduction. Antoine Culioli (qui répond aux questions d'A. Grésillon et J.-L. Lebrave) met en perspective l'entreprise énonciative qui a soutenu les premiers travaux des généticiens linguistes, alors même que sa conception de l'énonciation, conforme à ce que pouvaient penser les structuralistes positivistes (l'énonciation est la série d'opérations nécessaires à la construction d'un énoncé), se différencie de celle qu' a initiée Benveniste où le « je », impliquant immédiatement le « tu», est au centre de l'énonciation.

Un troisième entretien, autour de la bibliothèque de Jean-Claude Chevalier (interrogé par $V$. Chepiga et I. Fenoglio), présente une autre forme de genèse de la pensée linguistique, à l'échelle d'une vie, à travers la question des lectures constitutives du parcours d'un historien de la grammaire.

Ce dossier, relatif au "geste linguistique », donne à voir l'envergure d'un secteur qui avait échappé, jusque-là, à la critique génétique, et qui pourtant dès son ouverture offre un foisonnement de questions et de perspectives sur son domaine propre, mais aussi concernant l'observation contrastive des processus d'écriture en littérature et en sciences humaines. 